\title{
Saúde Mental em Medicina Geral Familiar - obstáculos e expectativas percecionados pelos Médicos de Família
}

\author{
Mental Health in General Family Medicine - \\ obstacles and expectations perceived by Family Physicians
}

Liliana Fernandes ${ }^{1}$

Nuno Basílio ${ }^{1}$

Sofia Figueira ${ }^{2}$

José Mendes Nunes ${ }^{3}$
${ }^{1}$ USF Carcavelos, ACES de Cascais. R. Tenente Coronel Melo Antunes. 2775 Cascais Portugal. josemendesnunes@ gmail.com

${ }^{2}$ USF S. Julião, ACES Lisboa Ocidental e Oeiras. Lisboa Portugal.

${ }^{3}$ Faculdade de Ciências Médicas, Universidade Nova de Lisboa. Lisboa Portugal.

\begin{abstract}
This study seeks to understand the difficulties experienced by family physicians (FP) in the management of mental disorders (MD) and their proposals to improve the quality of care. It is qualitative study with semi-structured interviews with ten family physicians. These were recorded, transcribed and their content analyzed. Eight thematic categories were identified: perceived working conditions and available resources; perceived level of training in mental health; therapies used for treatment of MD; mental health instruments used in consultation; MD addressed in Primary Health Care (PHC) and referral to hospitals; patient's reaction to referral; articulation of $\mathrm{PHC}$ with hospitals; proposals to improve mental health care in PHC. Articulation with the Mental Health Services suffers from lack of accessibility, one-way communication and delayed response. The FP propose creation of consultancies; multidisciplinary teams in the community; creating a two-way communication platform; continuous learning through discussion of cases. The FP have responsibilities in providing $M H C$. This requires working in a multidisciplinary team. Services should be organized to function as a learning system that allows the progressive improvement of the professionals and the improvement of the interfaces between them.
\end{abstract}

Key words Mental health, Primary health care, Ongoing medical education
Resumo Pretende-se conhecer as dificuldades sentidas pelos médicos de família (MF) na abordagem dos doentes com transtornos mentais (TM) e conhecer as suas propostas para melhorar os cuidados os cuidados de saúde mental (CSM). Estudo qualitativo. Realizaram-se entrevistas semiestruturadas e audio-gravadas a $10 \mathrm{MF}$. Com análise de conteúdo identificaram-se oito categorias temáticas: condições de trabalho percecionadas; formação em saúde; terapêuticas usadas para tratamento dos TM; instrumentos de saúde mental usados na consulta; TM abordados na atenção primária $(A P)$ e referenciadas a cuidados hospitalares; reação do doente à referenciação; articulação da atenção primária com a psiquiatria; propostas para melhorar os CSM na AP. A articulação com os serviços de saúde mental é deficiente pela falta de acessibilidade, comunicação unidirecional e atraso na resposta. Para melhorar os MF propõem criação de consultorias; equipes multidisciplinares; plataformas que permitam a comunicação bidirecional; aprendizagem contínua com a discussão de casos. O MF presta CSM, o que exige trabalho em equipe, com elementos da comunidade e dos hospitais. Os serviços devem organizar-se como sistemas aprendentes que permitam a progressiva melhoria dos profissionais e $o$ aperfeiçoamento das interfaces entre os mesmos.

Palavras-chave Saúde mental, Cuidados de saúde primários, Formação médica contínua 


\section{Introdução}

Segundo a Organização Mundial de Saúde (OMS), a Saúde Mental é o "estado de bem-estar em que o individuo tem consciência da sua capacidade de manter atividades produtivas, construir e manter relações gratificantes com outras pessoas, de se adaptar à mudança e de lidar com a adversidade" 1 .

Os transtornos mentais (TM) são um problema de Saúde Mundial, com consideráveis custos socioeconômicos e são uma das principais causas de incapacidade e de morbilidade ${ }^{2}$, sendo responsáveis por 5 das 10 causas de incapacidade.

Mundialmente, milhões de pessoas são afetadas por diversos $\mathrm{TM}^{3}$, que são responsáveis por uma média de 31\% dos anos vividos com incapacidade, sendo este valor mais alto na Europa, na ordem dos $40 \%{ }^{2}$. Tais valores poderão estar subestimados, dado que para além dos doentes com transtornos diagnosticáveis, existem muitos que não preenchem os critérios de diagnóstico de um TM, mas que mesmo assim beneficiam com a intervenção médica.

Segundo Parson, diversos fatores estão na origem dos TM, os quais interagem entre si e se manifestam através de alterações de interação interpessoal, de comportamento e de subjetividade pessoal, o que, em casos graves, é vivido como quase não sendo doença ${ }^{3,4}$. Contudo, até meados do século XX, os doentes sofriam em silêncio, estando sujeitos à discriminação, exclusão e estigmatização ${ }^{2}$, uma realidade que ainda se verifica atualmente por todo o mundo.

Os mitos sobre a doença mental e a estigmatização do doente ainda persistem, mesmo entre os profissionais de saúde, sendo ainda grande o desconhecimento sobre o progresso ocorrido nas últimas décadas relativamente ao diagnóstico e tratamento deste tipo de transtorno. Em muitos países, a Saúde Mental é ainda uma área negligenciada dentro do conjunto dos Serviços de Saúde, sendo o doente mental vítima de vários tipos de discriminação. No nosso país, os dados sobre prevalência são escassos. No entanto, os valores existentes apontam que não se afasta muito do encontrado na Europa ${ }^{2}$.

O diagnóstico baseia-se essencialmente na anamnese e muitos dos tratamentos baseiam-se na escuta e no diálogo com o doente. Os médicos devem desenvolver competências para entrevistar o doente, avaliar o seu estado mental e estabelecer uma relação terapêutica médico-doente efetiva, que permita identificar uma situação de risco no momento certo. O trabalho em saúde é de elevada exigência pelo seu impacto social, não bastando apenas tratar, mas igualmente prevenir e cuidar, para o que é fulcral desenvolver habilidades comunicacionais e estratégias para alcançar estes obetivos ${ }^{5}$.

A Saúde Mental (SM) é uma área diversificada, tendo por objeto o estudo dos estados cujos sinais e sintomas predominantes têm a ver com as emoções, as perceções, a cognição e a memória. Engloba os déficits de aprendizagem e os aspectos psicológicos. Na Atenção Primária (AP), a SM ocupa uma posição de relevo, sendo um dos principais motivos de consulta em Medicina Geral e Familiar (MGF), com uma prevalência de cerca de $50 \%{ }^{2}$. O médico deve estar atento para a possibilidade de existir um TM em qualquer pessoa, sendo capaz de reconhecer as suas características essenciais ${ }^{6}$ e assegurar a todos os usuários o acesso a Serviços de SM de qualidade ${ }^{2}$.

O Médico de Família (MF) é o primeiro ponto de contato do utente com o Serviço Nacional de Saúde, prestando cuidados de proximidade e promovendo a articulação com outras especialidades hospitalares ${ }^{3}$.

Através de uma abordagem holística, integrada e centrada na pessoa, o MF encontra-se numa posição que favorece o diagnóstico precoce de doentes com TM e assegurar atempadamente os cuidados necessários ${ }^{7}$.

Os Serviços de SM em Portugal têm um sistema de gestão e financiamento disfuncional no desenvolvimento e melhoria dos cuidados prestados. Existe uma fragmentação da resposta que não sustenta suficientemente a articulação entre a AP e os cuidados hospitalares, o que leva a uma perda na eficiência global. Além disso, persistem indefinições na aplicação das políticas de Saúde Mental, coexistindo diferentes modelos de articulação que, frequentemente, por serem superficiais ou mesmo mal desempenhados, não resistem com o tempo ${ }^{2,8}$.

Os últimos 20 anos têm revelado que mais de metade dos serviços de Psiquiatria desenvolveram programas de articulação com a Atenção Primária, com a realização de diferentes formas de consultoria, umas mais assentes na intervenção pontual (consultas), outras na formação e aprendizagem, tipo grupos Balint (em que os clínicos têm oportunidade de discutir e receber apoio sobre os seus casos difíceis). Contudo, para o sucesso terapêutico, mais importante que o vinculo institucional, é o clima de confiança e proximidade que se estabelece entre o doente e o $\mathrm{MF}^{2}$.

As características do Psiquiatra, de outros elementos da equipe de saúde mental, do Médico de 
Família, do interesse deste pela área da SM e das suas capacidades comunicacionais são condicionantes na articulação entre a atenção primária e os cuidados hospitalares. Trata-se de um processo complexo, faseado, com avanços e recuos, que exige esforço, reciprocidade e continuidade. Existe necessidade de melhorar esta articulação, sendo, por parte do MF, exigida uma rápida intervenção. Às equipes de SM, nos casos já sinalizados, exige-se informação de retorno. Por parte da atenção primária, espera-se que o MF faça uma correta discriminação dos doentes a referenciar. É necessário criar um projeto transversal, com definição das prioridades da atenção primária e dos cuidados hospitalares, sendo adaptado às realidades e necessidades locais, com qualidade na referenciação e informação de retorno ${ }^{2,9}$.

Para uma melhoria da prestação de Atenção de Saúde Mental, é fundamental determinar os obstáculos e as expectativas percecionados e experienciados pelos médicos de família quando abordam um doente com TM. Optou-se pela metodologia qualitativa dado que parece ser a que melhor permite responder a questões muito particulares, pois ocupa-se de uma realidade não redutível a números, permitindo aceder a um universo composto por significados, motivos, aspirações, crenças, valores e atitudes. O que corresponde a um espaço profundo das relações, dos processos e de fenômenos que não podem ser reduzidos à operacionalização de variáveis ${ }^{10}$.

O objetivo deste estudo foi o de compreender as dificuldades sentidas pelos médicos de família na abordagem dos doentes com transtornos mentais (TM) e conhecer as suas propostas para melhorar os cuidados em saúde mental.

\section{Métodos}

Foi realizado um estudo qualitativo, numa amostragem não probabilística, constituída por médicos especialistas em Medicina de Família, distribuídos pela maioria das Unidades do Agrupamento de Centros de Saúde (ACES) de Lisboa Ocidental e Oeiras. Para todas estas unidade de atenção primária os Serviços de Psiquiatria estão situados a menos $10 \mathrm{~km}$.

Todos os médicos contatados aceitaram participar do estudo e assinaram uma declaração de consentimento informado antes da entrevista. $\mathrm{O}$ número de unidades de análise foi determinado pelo princípio de saturação de informação, tendo sido realizadas 10 entrevistas. As entrevistas tiveram duração máxima de uma hora e obedeceram a um roteiro seguindo o método semidiretivo de entrevista. Foram feitas duas entrevistas piloto a fim de testar e aperfeiçoar o roteiro, treino da investigadora e elaboração da primeira grelha de sinopse. Com a experiência adquirida nas entrevistas piloto, foram introduzidas as correções consideradas pertinentes pela autora e pelos três validadores, um dos quais com experiência em metodologia qualitativa.

Após leitura interativa das entrevistas piloto e consideração das correções e as validações feitas pelos validadores com base nas mesmas, construiu-se uma grelha de sinopse.

As entrevistas foram audiogravadas e transcritas, sendo codificadas de forma a garantir a confidencialidade dos participantes.

O presente estudo foi autorizado pela Comissão de Ética para a Saúde da Administração Regional de Saúde de Lisboa e Vale do Tejo (ARSLVT), pela Direção Executiva e pela Direção Clínica do ACES de Lisboa Ocidental e Oeiras.

Todos os encargos relacionados com o estudo foram suportados pela primeira autora.

\section{Resultados}

Da análise das entrevistas, identificaram-se oito categorias temáticas: condições de trabalho percebidas e recursos disponíveis; nível de formação percecionado em saúde mental e conhecimento sobre terapêutica dos TM; terapêuticas usadas para tratamento dos TM; instrumentos de saúde mental usados na consulta; TM abordados em Atenção Primária e referenciadas aos cuidados hospitalares; reação do doente face à referenciação; articulação da Atenção Primária com os hospitais; propostas para melhorar os cuidados de saúde mental na comunidade.

No Quadro 1, são descritas as caraterísticas dos 10 médicos entrevistados.

Mais de metade dos participantes do estudo refere que os recursos na sua Unidade não são os ideais, variando as respostas entre muito escassos e adequados. Dois deles referem a presença de um psicólogo na sua Unidade. Três dos entrevistados mencionam um tempo de espera prolongado até à consulta de Psiquiatria.

A consulta de Psiquiatria demora algum tempo a ser respondida [E9;P1;L14-15]

Dois dos entrevistados identificaram dificuldades a nível da referenciação de alguns utentes por recusa do Serviço de Psiquiatria.

Todos os entrevistados consideram ter formação suficiente para lidar com este tipo de pro- 


\begin{tabular}{|c|c|c|c|c|}
\hline \multirow[t]{12}{*}{ 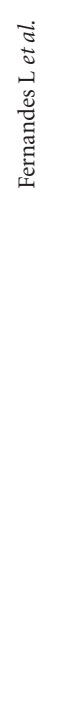 } & \multicolumn{4}{|c|}{ Quadro 1. Caraterísticas dos participantes do estudo. } \\
\hline & No & Gênero & Idade & Exercício profissional \\
\hline & 1 & F & 30 & 8 meses \\
\hline & 2 & F & 30 & 1 semana \\
\hline & 3 & $\mathrm{~F}$ & 55 & 26 anos \\
\hline & 4 & F & 61 & 31 anos \\
\hline & 5 & $\mathrm{~F}$ & 36 & 16 anos \\
\hline & 6 & $\mathrm{~F}$ & 63 & 33 anos \\
\hline & 7 & $\mathrm{M}$ & 36 & 7 anos \\
\hline & 8 & M & 41 & 10 anos \\
\hline & 9 & $\mathrm{~F}$ & 45 & 15 anos \\
\hline & 10 & $\mathrm{~F}$ & 36 & 6 anos \\
\hline
\end{tabular}

blemas. Um dos entrevistados refere haver "falta de formação" entre os profissionais de saúde, a qual, na sua opinião, conduz a estigmatização deste tipo de doentes nos Serviços de Saúde.

Eu acho que as pessoas não estão preparadas... os profissionais de saúde continuam a ter comportamentos que exprimem falta de formação em relação à doença mental. [E4;P1;L47-49]

A doença mental continua a ser estigmatizada em Portugal... as pessoas não estão sensibilizadas, encaram a doença mental como uma situação exclusiva. [E2;P1;L49-51]

Interrogados sobre o que seria preciso para se sentirem capazes de abordar este tipo de problemas, a maioria refere aprendizagem contínua baseada na discussão interpares (médico de família, psiquiatria, enfermagem, psicologia, assistente social, etc.) em estruturas de proximidade.

... onde se debatiam casos merecedores de uma segunda opinião, a opinião de um Psiquiatra... eram casos que nem sequer era preciso serem referenciados... conversávamos com ele, discutíamos e muitas vezes a solução aparecia de imediato. [E6;P1;L39-42]

... maior proximidade entre os dois cuidados de saúde, entre a equipe de saúde mental e a atenção primária, por exemplo, as consultadorias de saúde mental [E5;P2;L18-20]

Nove dos dez participantes referem ter conhecimento de terapêutica farmacológica na abordagem do doente com patologia mental. Quatro referem a psicoterapia, incluindo as intervenções breves.

... tento ajudar a pessoa a relativizar os problemas e a encontrar a solução. [E3;P1;L50-51]

Numa primeira fase, eu recuso a fazer farmacologia... tento que as pessoas tenham consciência, tento dar-lhes ferramentas para ajudar as pessoas a analisar e a compreender os problemas. [E4;P3;L13-15]

Um dos médicos refere a abordagem multidisciplinar e outro menciona terapia de suporte psicológico, negando efetuar psicoterapia.

Desde a psicoterapia, temos duas psicólogas... muitas vezes é acionada a enfermagem... [E7;P2;L16-18]

A psicoterapia não faço porque não sou psicoterapeuta... faço apenas algum suporte psicológico [E10;P2;L16-17]

Inquiridos em relação às terapêuticas utilizadas com maior frequência, todos referem a farmacológica. Os antidepressivos, nomeadamente os inibidores seletivos da recaptação da serotonina (ISRS) e os ansiolíticos, benzodiazepinas (BZD), são os fármacos mais usados. Alguns médicos referem recorrer a antipsicóticos e um deles o uso de neurolépticos.

Quanto às escalas de avaliação da patologia psiquiátrica, a maioria considera-as importantes e úteis. Contudo, referem não as usar no dia-adia por serem pouco práticas. Alguns deles mencionam não terem experiência na utilização das mesmas. Quando são utilizadas, são sobretudo por motivos didáticos.

... muitas vezes é para dar segurança a quem está ao meu lado, interno... [E1;P2;L18-19]

Questionados sobre a utilização destas, quatro dos entrevistados refere não as utilizar e os que recorrem a elas, fazem-no de forma irregular.

Não tenho por hábito abordar regularmente as escalas. [E5;P2;L48]

Abordados em relação às guidelines, a maioria considera-as como sendo importantes, mas não as usa.

Tal como qualquer guideline, acho que são orientações muito importantes e essenciais para nos proteger a nós e ao doente. [E1;P2;L23-24]

Três dos dez entrevistados consideram as guidelines pouco úteis, porque são limitativas e, em saúde mental, os doentes são mais variáveis.

As guidelines não podem ser interpretadas de forma tão rígida... num doente psiquiátrico não há propriamente uma quantificação... são mais regra de boa prática... devem ser mais flexiveis... não acho que sejam tão importantes. [E5;P2;L50-51]

Quando inquiridos sobre o conhecimento de critérios de referenciação, nove dos participantes referiram saber da sua existência. Grande parte considera serem importantes, estarem corretos e concordarem com eles. Dois referem não ter uma opinião formada sobre os mesmos. 
Todos mencionam as perturbações depressivas e a ansiedade ligeira moderada como as patologias predominantemente tratadas na atenção primária. A somatização foi referida por um dos médicos. Acham que as situações a tratar na atenção primária são sobretudo os problemas mais frequentes, incluindo aquelas sem necessidade de terapêutica farmacológica, dependendo do grau de gravidade e do estágio da doença.

... há situações que podem e devem ser tratadas por nós, sobretudo se tivermos alguma disponibilidade e predisposição para acolhermos pessoas sobretudo com depressão. [E4;P2;L34]

A doença bipolar, a esquizofrenia, a perturbação obsessivo-convulsiva e a personalidade borderline também foram consideradas como patologias passiveis de tratamento na atenção primária.

Quando interrogados sobre que patologias não referenciam ao hospital, mencionam as referidas anteriormente, e os doentes que recusam a referenciação para a Psiquiatria, ausência de ideação suicida e sintomas psicóticos, situações em que o controle sintomático é atingido, quando o acompanhamento pelo médico de família é suficiente e quando existe boa relação médicodoente.

...uma depressão reativa ou sem ser reativa, um luto mais complicado ou mesmo uma depressão endógena, numa pessoa que tenha um número de depressões recorrentes, mas que começamos cá a terapêutica e a resposta é positiva, bom acompanhamento, há uma boa ligação médico-doente, e por isso não sinto necessidade de referenciar. [E3;P3;L7-13]

No polo oposto, são referidas as situações a referenciar à psiquiatria como as depressões graves, ideação suicida, perturbações de personalidade, problemas de comportamento, dependências, psicoses, doença bipolar, esquizofrenia, recidivas, emergências e situações que constituam risco para o próprio e/ou terceiros. Referem dificuldades relacionadas com a terapêutica, sobretudo, necessidade de doses mais altas de medicação, mudança de terapêutica ou pouco conhecimento na sua utilização. Situações de agravamento do quadro clínico, necessidade de acompanhamento psicológico, má relação médico-doente, incapacidade de diagnóstico e o pedido do doente, são também consideradas por alguns dos participantes.

As respostas variam, desde a renitência e preferência pelo MF, por receio da estigmatização, com necessidade de envolvimento da família, em determinadas situações, à resposta adequada e positiva por parte do doente.
Acham que vão ser alvo de estigmatização, por serem referenciados a uma consulta de Psiquiatria. [E2;P2;L52-53]

A importância da comunicação é considerada por quatro dos participantes como sendo relevante na reação do doente, não sendo a decisão tomada apenas numa consulta.

Entre a relação estabelecida, explicando ao doente, informo que precisarei da ajuda dos colegas dos hospitais. [E7;P2;L28-30]

... cuidado de explicar o porquê da referenciação. [E9;P2;L38-39]

A experiência na relação com a psiquiatria transmitida pelos doentes aos seus médicos de família é variável. Referem que esta depende da relação estabelecida entre o terapeuta e o doente, havendo alguns que não conseguem estabelecer uma relação de empatia e, nestas condições, a experiência não é boa ou pouco satisfatória. Contudo, outros doentes referem uma boa relação com o psiquiatra.

O tempo de espera pela consulta de Psiquiatria é longo e a duração da consulta é insuficiente, particularmente porque são confrontados com uma realidade diferente da que estão habituados na atenção primária e pela polimedicação.

... a principal queixa que há é que eles não têm muitas vezes naquela consulta o tempo que têm na minha consulta... as consultas são curtas. E7;P3;L48-50]

Mais de metade dos participantes refere que a articulação com os hospitais não é a ideal, dois consideram-na inexistente e dois unidirecional, salientando a ausência de informação de retorno.

... não há articulação de volta, portanto não há verdadeira articulação porque é unidirecional. [E10;P3;L28-29]

... não é um trabalho de parceria, não é um trabalho conjunto... [E2;P4;L47]

Ausência de feedback dos colegas... [ E2;P4;L43]

Um dos médicos considera que a articulação é essencial e fundamental.

... que se articulassem e conversassem e, portanto, ver como nós nos podemos articular melhor... todos os serviços devem estar relacionados uns com os outros, nós todos temos de funcionar em rede... não sabemos funcionar em rede, estamos distantes uns dos outros. [E4;P4;L26-30]

Todos consideram a capacidade de resposta e o tempo de espera como sendo inadequados. $\mathrm{O}$ tempo de espera é prolongado, com má acessibilidade e ausência de resposta.

Quando referencio é porque acho que há mesmo necessidade de resposta... são situações que precisam de cuidados, normalmente não é uma coisa para daqui a dois meses. [E10;P3;L37-38] 
... raramente temos respostas dos colegas... não temos feedback... não há registos discriminados. [E6;P2;L39-41]

$\mathrm{Na}$ interação com a Psiquiatria, referem existir escassez de comunicação, sendo feita sobretudo através da referenciação por via informática que não permite a comunicação nos dois sentidos.

Não há canais de comunicação eficazes... poderia haver um email, um telefone... alguém que pensasse numa solução para um canal de comunicação dos profissionais... [E4;P4;L32-36]

... é um bocadinho difícil no dia-a-dia fazer a ligação para os hospitais, existem alguns entraves. [E1;P4;L15-16]

Quando questionados sobre os recursos desejados, referem que muita coisa pode ser feita para melhorar a interação entre estas duas especialidades. Sete dos entrevistados mencionam a realização da consultoria de Psiquiatria.

A questão das consultorias, como já houve no passado, se forem feitas de forma regular, também podem ajudar na resolução de alguns casos e até evitar algumas referenciações. [E3;P3;L32-34]

Foi mencionado o desejo da criação de um canal de comunicação que permitisse um contato mais direto, mencionando alguns deles exemplos para a criação do mesmo.

Maior possibilidade de comunicação, seja por via informática... há questões que podem ser esclarecidas se houvesse um canal de comunicação mais aberto. [E5;P3;L28-31]

Plataforma de comunicação... linhas telefónicas com acesso a um telefone mais direto. [E9;P3;L19-20]

O desejo de maior proximidade entre a atenção primária e os cuidados hospitalares é mencionado por grande parte dos entrevistados, propondo a criação de uma equipe multidisciplinar com formação na área e a elaboração conjunta de protocolos e guidelines.

... uma equipe multidisciplinar que tivesse formação na área, no acompanhamento de crianças em Pedopsiquiatria, no acompanhamento de adolescentes... que pudessem ser acompanhados pelas famílias, pelas escolas, pelos centros de saúde. [E4;P1;L19-23]

A presença de um psicólogo na unidade de saúde foi referida como um dos recursos desejados.

\section{Discussão}

As instalações deficientes e a carência de recursos, além de provocarem insatisfação, podem originar limitações na organização e na execução de todo o trabalho em Saúde Mental ${ }^{11}$. Contudo, os participantes apresentam uma opinião divergente em relação aos recursos disponíveis. Podese supor que a divergência encontrada reside no fato de, por exemplo, dois dos médicos terem na sua unidade de saúde a presença de um psicólogo, sendo percetível a satisfação dos mesmos relativamente ao trabalho efetuado por estes. A consultoria de psiquiatria, um dos recursos desejado por todos, é considerado como sendo a melhor via de contato entre os dois níveis de cuidados, permitindo aos médicos de família e psiquiatrias a orientação de situações e evitar referenciações desnecessárias para a psiquiatria. Este serviço, que em tempos já existiu em algumas destas Unidades e, proposto por Mitchell em 1983, ajuda o médico de família a reconhecer a morbilidade psiquiátrica e a referenciar no melhor tempo ${ }^{12}$.

Entre as experiências de integração a nível nacional, salientamos o exemplo de Grupo Integrado de Saúde Mental de Sintra que, sob a orientação do Professor João Sennfelt, teve início em 1985 e funcionou durante mais de 20 anos, em que através de reuniões multiprofissionais (psiquiatras de adultos e de crianças, médicos de família, assistentes sociais da Câmara Municipal e da Segurança Social, professores das escolas locais, etc.), nas quais se discutiam os casos identificados e se planeavam intervenções moldadas às necessidades de cada caso. As reuniões, para além da discussão e tomada de decisões terapêuticas, tinham forte componente formativo em que todos aprendiam com todos e o próprio Grupo aprendia e aperfeiçoava as suas intervenções ${ }^{13}$, fomentando a partilha de informação e a comunicação entre os dois níveis de cuidados. Os entrevistados consideram que este formato tem a vantagem de permitir conhecer melhor o Psiquiatra a quem referenciam, melhorar a clareza do pedido e ter maior brevidade na obtenção de resposta. É também uma via propícia à aquisição de conhecimentos e novas competências na abordagem dos transtornos mentais. O Psiquiatra consegue ter um contato mais próximo com a medicina e com os problemas da atenção primária. $\mathrm{O}$ doente mental beneficia de acesso mais fácil, cuidados inseridos na comunidade, maior celeridade em tratamentos especializados e continuidade de cuidados.

Neste sentido, os médicos propõem a presença de uma equipe multidisciplinar com formação 
na área de saúde mental, que atue na comunidade, mais próxima dos doentes e seus familiares. Esta deveria estar estreitamente articulada com a atenção primaria, valorizando o setor social e as organizações não governamentais ${ }^{14}$, o que permite que os problemas de saúde mental não estejam centrados no hospital e as consultas serem feitas predominantemente na comunidade ${ }^{3}$. Os cuidados seriam prestados num meio menos restritivo, devendo ser coordenados e integrados de forma a facilitar a continuidade de terapêuticas, com um leque de possibilidades e programas capazes de responder às necessidades em saúde mental da população, devendo os familiares serem considerados como parceiros fundamentais na prestação de cuidados.

Os médicos entrevistados defendem que a articulação eficiente exige melhorar e promover a comunicação entre o médico de família e o psiquiatra, pelo que propõem a criação de uma plataforma que permita a comunicação entre as especialidades. Afirmam que atualmente a comunicação de referenciação existente é unidirecional que nem prevê informação de retorno do psiquiatra. Quando existe retro informação, é feita por carta ou através do sistema informático alternativo. Por outro lado é fulcral a criação de oportunidades de discussão de casos, onde se dê a partilha de saberes com a consequente formação, isto é, um sistema que seja autoaprendente.

Apenas um dos entrevistados declara haver "falta de formação" dos profissionais de saúde, o que determina estigmatização. Os restantes dizem não observar situações de estigmatização nas suas unidades de saúde, mas têm opinião sobre as suas consequências.

Apesar de todos considerarem terem formação suficiente, nove propõem como formas de melhorar a sua abordagem uma formação e aprendizagem contínua, feita, principalmente, com base na discussão de casos clínicos partilhados com o psiquiatra e outros profissionais de saúde, sobretudo devido à plasticidade dos distúrbios mentais e pelo fato de que a experiência só se adquire com a prática clínica, o que implica ação e modelagem de atitudes ao longo da vida profissional.

$\mathrm{Na}$ abordagem terapêutica, alguns médicos referem fazer sempre a psicoterapia. Contudo, a resposta à questão formulada sobre as terapêuticas mais utilizadas mostra incoerências, dado que referem os psicofármacos, não mencionando a psicoterapia, sobretudo os que referiram utilizarem-na sempre. Isto levanta a hipótese de que a psicoterapia não é levada a sério, não é reconhe- cida como terapêutica ou então apenas é referida no sentido de dar uma resposta politicamente correta. Esta desvalorização ou a sua mistificação é revelada quando um dos participantes nega que "faz psicoterapia" para dizer que "faz suporte psicológico”. Entre a terapêutica farmacológica mais utilizada, os antidepressivos são os mais usados, sobretudo os Inibidores seletivos da recaptação da serotonina (ISRS). Há clínicos que dizem usar uma diversidade de moléculas, pertencentes a classes diferentes, o que evidencia a liberdade de escolha dos mesmos para o tratamento destas patologias.

É consensual a elevada prevalência da patologia mental na atenção primária ${ }^{2}$. O médico de família assume uma importância acrescida na prestação de cuidados na área da saúde mental. Contudo, existem dificuldades no diagnóstico destas patologias, e há situações que podem não ter sido registadas por incapacidades na abordagem $^{4}$. O fato de lidarem com todo o tipo de situações e patologias, não só da área psiquiátrica, pode revelar-se um entrave. Todos mencionam as perturbações depressivas e a ansiedade ligeira a moderada, apenas um menciona a "somatização", não sendo possível saber se o conceito é relativo à clássica somatização ou se também inclui os sintomas somatoformes. O limite da referenciação é a ausência da melhoria com a intervenção do médico de família. As patologias que aludem referenciar à psiquiatria são complementares às que não referenciam.

As escalas de avaliação, guidelines e critérios de referenciação são considerados como sendo instrumentos importantes. Contudo, são pouco utlizados. Questiona-se se esta situação está relacionada com a sobrecarga de trabalho, dispêndio de tempo de consulta que estes doentes exigem face às exigências expostas ou se as consideram de utilidade reduzida.

A reação do doente à proposta de referenciação pode depender da personalidade, do receio da estigmatização, ou por inadequadas aptidões de comunicação do médico de família. Estes são fatores que podem facilitar ou criar resistências à referenciação.

A prevalência dos transtornos mentais é elevada e o seu impacto é muito grande no nível dos indivíduos, da família e da sociedade. O médico de família contata com a maioria dos doentes que sofrem TM, sendo responsável pela identificação, diagnóstico, manuseio e referenciação destas patologias. Contudo, para que tal seja possível, o acesso à informação acerca dos recursos disponíveis nesta área é essencial. Mas, os serviços de 
saúde mental apresentam insuficiências de acessibilidade, de equidade e na qualidade de cuidados.

Os benefícios da integração da saúde mental na atenção primária são reconhecidos por todos os médicos como importantes, dado que essa integração assegura a acessibilidade da população em geral aos cuidados hospitalares, facilita a prestação de cuidados de forma holística e centrada na pessoa, e minimiza o estigma, permite uma maior gestão do tempo e evita a saturação dos hospitais. Para que tal seja possível propõem essencialmente quatro medidas: a existência de um serviço de consultoria; a presença de uma equipe multidisciplinar com formação em saúde mental na comunidade, constituída por vários profissionais, como psicólogo, enfermeiro, assistente social, etc.; a criação de uma plataforma de comunicação que permita a partilha de informação; e uma aprendizagem contínua. Isto permitirá que o médico de família, o psiquiatra e outros profissionais trabalhem em conjunto, potenciando a melhoria da continuidade de cuidados, ajudando o médico de família na identificação da morbilidade psiquiátrica e na definição do melhor momento para referenciar. Para além disso, o médico de família conhece melhor o especialista a quem pode referenciar os seus utentes, com maior clareza do pedido e celeridade na resposta, constituindo-se através da discussão de casos, uma via propícia à aquisição de novos conhecimentos e capacidades em saúde mental, assim como a partilha de saberes, garantindo desta forma uma formação e aprendizagem contínuas.

Assim, a articulação entre os médicos de família e os psiquiatras é o caminho para a prestação de cuidados adequados, em que os diferentes profissionais trabalhem em conjunto, sendo longo o caminho a percorrer e o trabalho a desenvolver.

\section{Limitações do Estudo}

O presente estudo foi realizado em unidades prestadoras de atenção primária pertencentes ao ACES de Lisboa Ocidental e Oeiras. Foi realizado com médicos especialistas em medicina de familia, que têm a seu cargo uma lista de utentes e que não desempenham funções de coordenação. Apenas um dos entrevistados se mostrou descontente com o modo e com o conteúdo do roteiro da entrevista. Dois deles, após conclusão da mesma, referiram não se sentirem à vontade por estarem com receio de serem alvo de juízo de valores.

Todas as entrevistas decorreram no consultório médico dos participantes. Apesar de todas terem decorrido com horário marcado, não ultrapassando o tempo previsto, três delas foram interrompidas por fatores externos, tais como funcionários das unidades e chamadas telefônicas.

\section{Propostas de Investigação para o Futuro}

Após a realização deste estudo qualitativo, outros podem ser desenvolvidos. Por exemplo, a aplicação de um questionário a utentes com patologia mental com o objetivo de conhecer a forma como vêm o médico família a prestar cuidados de saúde mental, quais são as suas expectativas sobre o comportamento que profissionais e os serviços devem ter quando lhes prestam cuidados. Da mesma forma, um estudo qualitativo poderia ser realizado no sentido de conhecer a perceção dos Psiquiatras acerca da comunicação com os médicos de família. Clarificar o que os médicos de família entendem por psicoterapia e que tipo de formação precisam para acreditarem no seu valor e se sentirem confiantes na abordagem da vertente psicológica dos doentes. 


\section{Colaboradores}

L Fernandes, N Basílio, S Figueira e JM Nunes contribuíram nas várias fases do trabalho excepto na recolha de dados, tendo sido feita apenas pela autora L Fernandes.

\section{Referências}

1. Organização Mundial de Saúde. Definição de Saúde Mental. [Internet]; 2016 [cited 2016 Mar 18]. Available from: http://www.who.int/mediacentre/factsheets/ fs200/en/

2. Portugal. Restruturação e Desenvolvimento dos Serviços de Saúde Mental em Portugal. Plano de Ação 2007-2016. Lisboa: Comissão Nacional para a Restruturação dos Serviços de Saúde Mental em Portugal; 2006

3. Portugal. Relatório sobre a Saúde no Mundo 2001. Saúde Mental: Nova Concepção, Nova Esperança. Lisboa: Direcção Geral da Saúde; 2001

4. Oliveira J. Desvio, Rótulo e Estigma na Doença Mental. Plataforma Barómetro Social. [4a série de 2014 de Artigos de Opinião]. 2014. [cited 2014 nov 17]. Available from: http://www.barometro.com.pt/2014/12/23/desvio-rotulo-e-estigma-na-doenca-mental

5. Macedo AF, Azevedo MHP. Relação Médico-Doente. 2011. [cited 2015 Mar 5]. Available from: http://www. fspog.com/fotos/editor2/cap_04.pdf

6. Harrison P, Geddes J, Sharpe M. Psiquiatria - Guia Prático de Medicina. Lisboa: Climepsi Editores; 2006

7. Sarmento CM. Referenciar um doente: Quem, como, quando e porquê?. Psiquiatria de Ligação com os Cuidados de Saúde Primários. Acta Médica Portuguesa 1997; 10(5):3339-3344

8. Direção Geral da Saúde. Rede de referenciação de Psiquiatria e Saúde Mental. Lisboa: Direção Gral da Saúde; 2004. [cited 2015 Mar 20]. Available from: http:// www.dgs.pt/upload/membro.id/ficheiros/i007439.pdf

9. Associação de Apoio às Pessoas com Perturbação Mental Grave. Guia de Recursos Humanos em Saúde Mental. Edição Encontrar+se. [cited 2014 Dec 10]. Available from: http://www.anarp.org.pt/lib/pdfs/centroreabilitacao/publicacoes/Guia_de_Recursos_em_ Saude_Mental.pdf

10. Guimarães JMX, Jorge MSB, Assis MMA. (In)satisfação com o Trabalho em Saúde Mental: Um Estudo em Centros de Atenção Psicossocial. Cien Saude Colet 2011; 16(4):2145-2154.

11. Allen J, Gay B, Crebolder H, Heyrman J, Svab I, Ram P, Evans P. A Definição Europeia de Medicina Geral e Familiar (Clínica Geral/Medicina Geral e Familiar). Revista Portuguesa de Clínica Geral 2005; 21:511-516

12. Brasil. Ministério da Saúde (MS). Saúde Mental e Cuidados de Saúde Primários. Coordenação Estratégica para os Cuidados de Saúde Primários. Lisboa: Ministério da Saúde; 2011. [cited 2014 Dec 10]. Available from: http://www.acss.min-saude.pt/Portals/0/saude_mental_e_gcs_em_csp_2011.05.25_2.pdf

13. Rebelo MF, Gonçalves MJ, Bolina FM. A $3 a$ Face da Lua - Saúde mental em Cuidados de Saúde Primários. 2a ed. Loures: Edições Lusodidacta; 2015.

14. Portugal. Coordenação Nacional para a Saúde Mental. Plano Nacional de Saúde Mental 2007-2016, Resumo Executivo. Lisboa: Coordenação Nacional para a Saúde Mental; 2008

Artigo apresentado em 18/09/2016

Aprovado em 05/12/2016

Versão final apresentado em 07/12/2016 
